\title{
Verbal labeling as an assimilation mnemonic for abstract visual stimuli: The sample case of recognition memory for Chinese characters
}

\author{
PAUL VERHAEGHEN, TIBOR PALFAI, and MICHAEL P. JOHNSON \\ Syracuse University, Syracuse, New York
}

\begin{abstract}
In four experiments, we examined the effect that presenting a verbal stimulus (viz., an English noun) alongside an abstract visual stimulus (viz., a Chinese character) enhances recognition memory for the abstract visual stimulus. Experiment 1 demonstrated that the character-plus-word combination at both encoding and retrieval results in better recognition than does a character-alone presentation or presenting the combination at encoding only. Experiment 2 demonstrated that presenting the word first and then the character results in better performance than does the opposite order. Experiment 3 showed that the concreteness value of the word, not familiarity, is the critical factor. In Experiment 4, presentation time was varied. More time was needed for liftoff from chance level for the word-character combination than for the character-alone presentation. Together, the results suggest that subjects spontaneously assimilate stimulus and word into a single representation by building asymmetric effortful imagery associations, going from the English word to the Chinese character.
\end{abstract}

What makes a stimulus memorable? One of the bestestablished findings in the field is that access to a deep representation of a stimulus - that is, the extraction of a stimulus's meaning, as opposed to its perceptual characteristics-enhances recall and recognition considerably (see, e.g., Craik \& Tulving, 1975). But what about stimuli that do not carry meaning - that is, stimuli that are all perceptual characteristics, all abstract shapes? In one earlier study (Verhaeghen et al., 2000), we investigated memory for one such type of stimulus - namely, memory for Chinese characters in non-Chinese readers - and contrasted this with memory for words denoting objects and living things. Our paradigm of choice was a time-accuracy paradigm (i.e., encoding time was varied, with a maximum of $6 \mathrm{sec}$ per stimulus); we tested recognition through a two-alternative forced choice procedure. Figure 1 reproduces the results. It is clear that in this group of Englishspeaking readers, memory for Chinese characters was worse than memory for English words at all presentation times. The data also strongly suggested that a further increase in presentation time will not alleviate the difference between the two classes of stimuli. This suggests that the

An earlier version of this article was presented at the 43rd Annual Meeting of the Psychonomic Society, Kansas City, MO, November 2003. We thank Marc Howard for making useful comments on a draft version of the manuscript. Correspondence concerning this article should be addressed to P. Verhaeghen or T. Palfai, Department of Psychology, Syracuse University, 430 Huntington Hall, Syracuse, NY 13244-2340 (e-mail: pverhaeg@psych.syr.edu or palfai@psych.syr.edu).

Note-This article was accepted by the previous editorial team, when Colin M. MacLeod was Editor
Chinese characters are represented much more poorly in long-term memory. In other words, there appears to be a real limit to the amount of information that can be retained from complicated, abstract stimuli.

One manipulation that has been found to enhance recognition memory for unfamiliar, meaningless, and complex stimuli is verbal labeling (e.g., Daniel \& Toglia, 1976; Ellis, 1968; Ranken, 1963; Santa, 1975). In these experiments, a complex, random shape is associated with a verbal label, sometimes random as well (e.g., Daniel \& Toglia's subjects associated polygons with colors), but often not (e.g., Santa used labels suggestive of the shapes in one of the conditions), and this association leads to better performance on a subsequent (mostly forced choice) recognition test.

At least two types of hypotheses have been advanced to explain why presenting a verbal label along with an abstract or meaningless shape might enhance one's recognition memory for the shape. The oldest of these originates in Gestalt psychology (e.g., Carmichael, Hogan, \& Walter, 1932) and has been labeled the assimilation hypothesis (Daniel \& Toglia, 1976). This hypothesis asserts that during the encoding episode, the abstract stimulus and its verbal label become assimilated into a single configuration and that this integrated configuration is what is retained in memory.

In contrast to this associative view, the hypotheses put forward in the 1960s and 1970s state that the label serves merely to enhance encoding of the stimulus. For instance, the conceptual-coding hypothesis (Ellis, 1973) maintains that labels have their effect by directing the subject's attention to particular attributes of the stimulus. Evidence for this position has come from experiments using random 


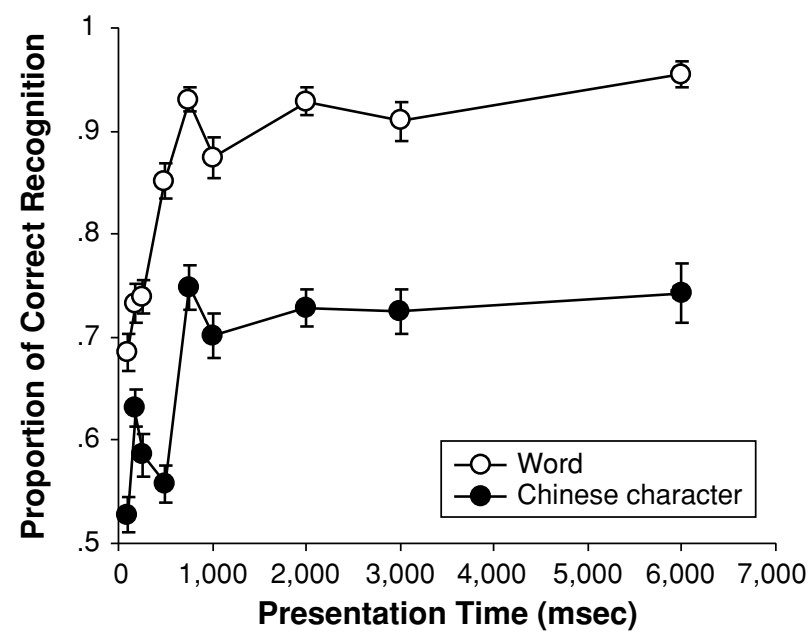

Figure 1. Time-accuracy curves for recognition memory for concrete words and Chinese characters in a previous study (after Verhaeghen et al., 2000, used by permission).

polygons that had relevant verbal associates, as determined by previous studies (e.g., Ellis, 1968). In these experiments, recognition memory was enhanced only when a random shape was paired with its relevant associate. Irrelevant verbal labels failed to elevate recognition memory above the level obtained by meaningless labels (viz., CVC trigrams). The suggestion is that the relevant labels capture a distinctive aspect of the stimulus; presenting such a label then draws attention to that aspect of the stimulus, which makes it more memorable. Another incarnation of the enhancedencoding hypothesis is the integration hypothesis (Santa, 1975), which states that labeling a complex stimulus serves to integrate its parts into a single coherent representation by promoting internal associations. Evidence for this position has come from a redintegrative memory task, in which the subject is presented with part of the stimulus and has to recognize the rest. Labeling boosts performance on the redintegration task.

Interestingly, the debate in the 1960s and 1970s seemed to have simply disregarded the assimilation hypothesis, focusing instead on the different instantiations of the stimulus-encoding view. The assimilation view, however, seemed to us a very good candidate mechanism for explaining the verbal-labeling effect. After all, associations, especially visual associations, are a traditional device for memory enhancement, as work on time-honored mnemonic devices such as the method of loci and the pegword mnemonic testifies (for an overview, see Bellezza, 1983). These mnemonics pair unrelated stimuli (i.e., a set of places and a set of objects, respectively) with the wordsto-be-remembered, and this leads to remarkable increases in performance (for a meta-analysis, see Verhaeghen, Marcoen, \& Goossens, 1992). Verbal-labeling studies are not completely analogous to visual mnemonics studies, if only because the stimuli are abstract shapes, rather than words, and the associates are single nouns not forming a series, but the underlying mechanism may well be identical.
The aim of the present article is then to revisit the issue of verbal labeling of abstract stimuli. In line with our previous work, we used Chinese characters as stimuli and native speakers of English with no knowledge of Chinese as subjects. We will present the results from four experiments reexamining the assimilation hypothesis. In the first experiment, we established the necessary character of the association between the verbal label and the Chinese character. In the second experiment, we investigated the direction of the association. In the third experiment, we investigated the nature of the association, and in the fourth experiment, we investigated its time course. We should note here that in order to provide a fair assessment of the assimilation hypothesis, our subjects remained largely uninstructed; that is, apart from explaining the memory task and the different conditions of the experiments, we did not refer at any time to the possibility of building associations. The cognitive processes leading to the formation of assimilative associations as observed here were, therefore, initiated by the subjects themselves.

\section{EXPERIMENT 1}

The general design of our experiments was to pair each of the to-be-studied Chinese characters with a verbal label. The hypothesis was that providing this label would aid recognition of the character by forming an association between selected features of the character and the meaning of the word; this is the assimilation hypothesis. The alternative hypothesis is that the verbal label serves solely to enhance encoding. To investigate these hypotheses, we examined pairwise forced choice recognition for lists of Chinese characters under three conditions: (1) a condition in which the character was presented alone during encoding, (2) a condition in which the character was presented along with a concrete noun during encoding but no nouns were presented during the recognition phase, and (3) a condition in which the character was presented along with a concrete noun during encoding and that noun was presented during the recognition phase, along with the corresponding target character and a distractor character. If the stimulus-encoding hypothesis is correct, presenting the label with the Chinese character during encoding should suffice to increase recognition performance. If the assimilation view is correct, it might be necessary to present the verbal label at retrieval, as well as at encoding. It is possible that both views are correct, and then we would expect three distinct levels of recognition: Chinese characters presented without a label would yield the lowest level of recognition, character plus word at encoding and retrieval would yield the highest level of recognition, and character plus word at encoding only would result in an intermediate level of recognition.

As was stated above, in order not to bias the subjects toward assimilative encoding, we decided not to instruct them to use the label in any way. We also decided to make the labels random; that is, the words we used were concrete nouns not associated in any way to the shape of the Chinese character (as judged by the authors and our re- 
search assistants) or to the meaning it denoted. The alternative would be to use labels that somehow would be suggestive of the shape of the character. Some researchers have, indeed, used such descriptive labels (e.g., Ellis, 1968; Santa, 1975), but a number of studies in which ambiguous shapes have been used have demonstrated that descriptive labels can lead to verbal overshadowing; that is, the memory trace for the shape gets tainted with the label, leading to reduced accuracy on subsequent recognition trials (e.g., Carmichael et al., 1932; for a meta-analysis on the verbal-overshadowing effect with faces, see Meissner \& Brigham, 2001).

\section{Method}

Subjects. The subjects were 35 undergraduate students, who received course credit for their participation.

Materials and Procedure. For the Chinese character stimuli, we used the same pool of 400 characters as that in Verhaeghen et al. (2000). These consisted of characters selected from a Chinese font package (UnionWay AsianSuite). Early piloting showed that characters that were very complex (typically consisting of 20 or more different strokes or containing more than two distinct parts) were very hard to recognize. Consequently, the characters selected for the study were screened for relative simplicity as visual stimuli. Screening was done by the authors. All the characters consisted of at least 4 strokes and no more than 20 strokes. Four random examples of characters are shown in Figure 2.

The pool of words consisted of 400 nouns selected from the MRC Psycholinguistic Database, also used in Verhaeghen et al. (2000). The words were selected to be both familiar and concrete/imageable. Words were between three and five letters long and had a score of 500 or higher on both the concreteness and the imagery scales as devised by the MRC (these scales range from 100 to 700 and are a merging of the norms by Gilhooly \& Logie, 1980, Toglia \& Battig, 1978, and an unpublished expansion of the norms by Paivio, Yuille, \& Madigan, 1968). Average familiarity rating of the stimuli was 508 $(S D=67)$ on a $100-700$ scale .

Three conditions were implemented in a within-subjects design; order of condition was counterbalanced across subjects. Presentation during encoding was listwise, as was presentation during the retrieval phase. Recognition was pairwise (one foil, one target) and forced choice. In the first condition, only Chinese characters were shown during encoding and retrieval. In the second condition, a word was paired at random with each character during encoding only. In the third condition, a word was paired at random with each character at encoding; the same word was then shown with its associated character and a foil at retrieval. For each condition, three lists were presented, for a total of nine lists. Presentation of each list started with a keypress initiated by the subject. Each list consisted of 19 targets (presented at encoding) and 19 foils (each paired with a target at retrieval); targets and foils were drawn at random without replacement from the pool of 400 stimuli. The stimuli were projected one at a time onto the center of a 13 -in. monitor. Chinese characters filled a 5-cm-square view port at the center of the screen. Words were presented in lowercase letters of a bold Times-like font (48 points), about $17 \mathrm{~mm}$ high, immediately above the character. The subjects were allowed to adopt a viewing distance from the screen that they considered most comfortable. The 19 study items of a list were presented sequentially at a rate of $3 \mathrm{sec}$ per item, with an interstimulus interval of 0 . The Verhaeghen et al. (2000) study has shown that $3 \mathrm{sec}$ is sufficient to elicit maximum performance in a Chinese-character-only condition. The subjects were instructed that a two-alternative forced recognition test would follow presentation, and the presence or absence of the word during retrieval was announced prior to presentation of the stimulus list. The three lists for each condition were presented contiguously.

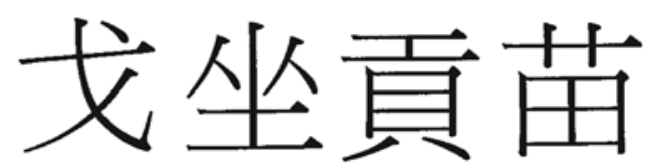

Figure 2. Sample Chinese character stimuli.

Immediately after presentation of each list, recognition trials began. Two Chinese characters, one target and one foil, were presented simultaneously on the screen, side by side, and the subjects responded by pressing a key (either $\mathrm{z}$ or /) that corresponded to the location of the stimulus they recognized as having been presented during encoding. The order of presentation of the stimuli within each list during retrieval did not correspond to the order of presentation within the list during encoding. Accuracy, rather than speed of responding, was encouraged. For the condition in which the words were shown during the recognition trials, each word was projected onto the screen immediately above the two characters.

The subjects were tested individually in a quiet, comfortable, light-sealed room with controlled ambient lighting. All assessments were taken in a single session, typically lasting about $40 \mathrm{~min}$. The subjects were encouraged to take breaks whenever they felt tired; few subjects, however, chose to take breaks.

The alpha level for this and all the subsequent experiments was set at .05 .

\section{Results}

The results are depicted in Figure 3. All the conditions yielded recognition performance significantly better than chance (i.e., 50\% accuracy). We conducted an ANOVA with condition and order of presentation within condition (to test for practice or interference effects) as withinsubjects variables. We found a significant main effect of condition $\left[F(2,33)=37.96, M S_{\mathrm{e}}=0.012\right]$. Neither the main effect of order $\left[F(2,33)=1.08, M S_{\mathrm{e}}=0.009\right]$ nor its interaction with condition $\left[F(4,31)=1.40, M S_{\mathrm{e}}=\right.$ $0.008]$ reached significance. Bonferroni tests for pairwise comparisons indicated that the presence of a word at both

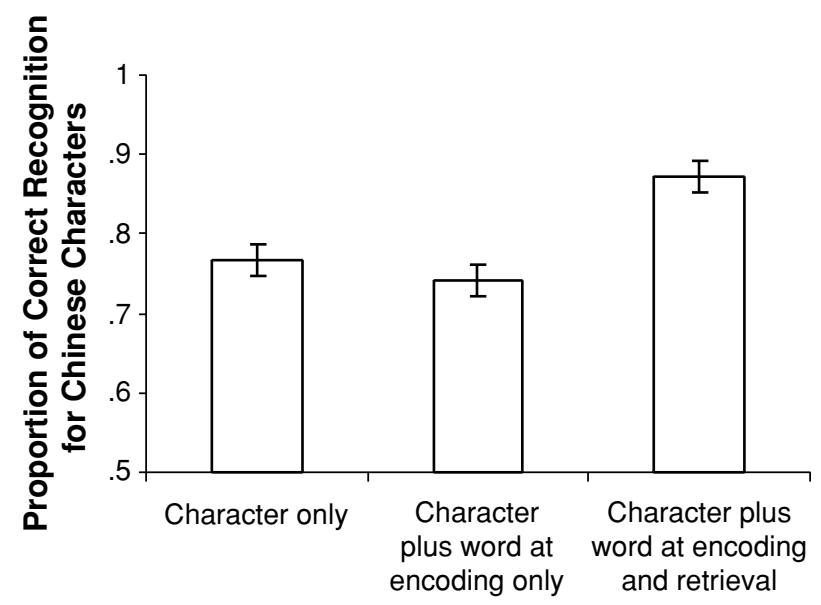

Figure 3. Results from Experiment 1. Recognition memory for the Chinese characters was highest when paired with words at encoding and retrieval. Presenting a word during encoding only did not enhance recognition memory for the character. Error bars denote $\pm 1 S E$. 
encoding and retrieval reliably enhanced recognition, in comparison with the two other conditions $(p<.000$ for both comparisons). For the latter two conditions, recognition was similar $(p=.17)$.

\section{Discussion}

Our results conform to the predictions of the assimilation hypothesis. With this set of stimuli, Chinese characters and random verbal labels, we did not find that presenting the label at encoding improved recognition $(74 \%$ vs. $77 \%$ in the baseline condition in which the character was presented alone; guessing level was at 50\%). This finding goes against any form of the enhanced-encoding hypothesis. We did find, however, that presenting the noun with the character at encoding as well as at retrieval did boost performance, to $87 \%$ correct. This indicates that an association has been formed between the label and the character; presenting the label then makes the association retrievable and, hence, the character itself. We take this as evidence for the assimilation view. If the label guided and enhanced encoding by helping the subject to find relevant or distinctive features in the character, the mere presence of the character should be sufficient to improve recognition. Note that our result does not imply that verbal labels will always fail to guide encoding (the Ellis, 1968, study suggests that more descriptive labels might have worked for that purpose) but, rather, that the assimilation mechanism can operate in the absence of any encoding enhancement or, at least, an encoding enhancement that is memorable enough to influence a forced choice recognition test.

One reviewer objected that our finding might be merely an instance of the encoding specificity principle (e.g., Tulving \& Thomson, 1973). Encoding specificity entails that reinstating the encoding context works as a retrieval cue and, thereby, improves recognition memory. We would maintain that even the existence of a mere encoding specificity effect, in fact, affirms the assimilation hypothesis: The effect shows that during encoding, the character has been linked with the word to the degree that the word works as an effective retrieval cue for the character. One other result, however, strongly suggests that the effect is more than merely a context effect. Skipping ahead to Experiment 4 , we will find there that forming the association takes additional time. This suggests that the label does not function merely as a context but that the associations are built in a deliberate, time-consuming manner.

We also note that order of presentation within condition did not yield a significant effect. This indicates either that learning or interference effects were minimal or that learning and interference canceled each other out.

\section{EXPERIMENT 2}

Having shown that verbal labels build associations, rather than merely enhancing encoding, we designed Experiment 2 to investigate the direction of the association. Our working assumption was that assimilation entails the formation of associations between selected features of the character and the semantic content of the word. The feature extraction process can take at least two forms. First, it is possible that features are extracted that are associated with the semantic content of the label. For instance, if the word presented is clown, the subjects would first access the meaning of the word and then search the Chinese character for something clownlike, thereby building a strong association. An alternative hypothesis is that the subject is not guided by the meaning of the word but extracts distinctive features from the Chinese characters; it would be those features that would be associated with the word. (Note that these features need not be identical across subjects, but they might be intraindividually stable.) The latter mechanism is similar to what happens in mnemonics, such as with the method of loci or the pegword mnemonic. If the semantic associates interpretation of the assimilation phenomenon is correct, it would follow that the association will be asymmetric. Knowing the meaning of the word during presentation of the Chinese character might aid with isolating and extracting particular features for association, whereas not knowing the meaning of the word during presentation of the character would not be of benefit to the subject. If the distinctive features hypothesis is correct, the features extracted for any stimulus would be independent of the meaning of the word. Under this hypothesis, therefore, the association should be symmetric; it would not matter whether the label or the character was shown first.

We tested these hypotheses by including three conditions - namely, two sequential conditions and one simultaneous condition: (1) a condition in which the word was presented first and then the character, (2) a condition in which the character was shown first and then the word, and (3) a comparison condition in which the word and the Chinese character were presented simultaneously. The contrast of interest was between the first and the second of these. If the semantic associates hypothesis is correct, we would expect a considerable difference between the wordfirst condition and the character-first condition. If the distinctive feature hypothesis is correct, we would expect equivalent effects across the two sequential conditions. The simultaneous presentation condition was included as a reference. One decision to make about this condition was the presentation time. The two stimuli could be presented either for the time the character was on screen in the sequential conditions or for the total combined time of the label and the character in the sequential conditions. We decided to use the latter. This potentially biased the simultaneous condition toward higher performance, because the Chinese character was now on screen for twice the amount of time than in either of the sequential conditions (and so was the label).

\section{Method}

Subjects. The subjects were 43 undergraduate students, who received course credit for their participation.

Materials and Procedure. The stimulus pool for the present experiment was identical to the pool used in Experiment 1.

Three conditions were used in a within-subjects design; order of condition was counterbalanced across subjects. In one condition, 
first a Chinese character was shown and then the corresponding word (with no overlap of stimuli on the screen). In another condition, first a word was shown and then the corresponding character (with no overlap of stimuli on the screen). In yet another condition, the word and the Chinese character were shown simultaneously. Presentation time was $3 \mathrm{sec}$ for each word or character. Details of list and stimulus presentation were identical to those in Experiment 1.

Immediately after presentation of each list, recognition trials began. Two stimuli, one target and one foil, were presented simultaneously on the screen, side by side, and the subjects responded by pressing a key (either $\mathrm{z}$ or /) that corresponded to the location of the stimulus they recognized as having been presented during encoding; the corresponding word was projected directly above the two characters. The order of presentation of the stimuli within each list during retrieval did not correspond to the order of presentation within the list during encoding.

The subjects were tested individually in a quiet, comfortable, light-sealed room with controlled ambient lighting. All assessments were taken in a single session, typically lasting about $40 \mathrm{~min}$. The subjects were encouraged to take breaks whenever they felt tired; few subjects, however chose to take breaks.

\section{Results}

The results are depicted in Figure 4. All the conditions yielded recognition performance significantly better than chance (i.e., 50\% accuracy). An ANOVA with condition and order of presentation within condition (to test for practice or interference effects) as within-subjects variables yielded a significant main effect of condition $\left[F(2,41)=7.62, M S_{\mathrm{e}}=0.016\right]$. Neither the main effect of order $\left[F(2,41)=1.09, M S_{\mathrm{e}}=0.005\right]$ nor its interaction with condition $\left[F(4,39)=1.43, M S_{\mathrm{e}}=0.006\right]$ reached significance. Bonferroni tests for pairwise comparisons indicated that presenting the character before the word yielded lower recognition, in comparison with the two other conditions ( $p=.011$ and .001 for word before character and for simultaneous presentation, respectively). The latter two conditions yielded statistically equivalent levels of recognition $(p=.25)$.

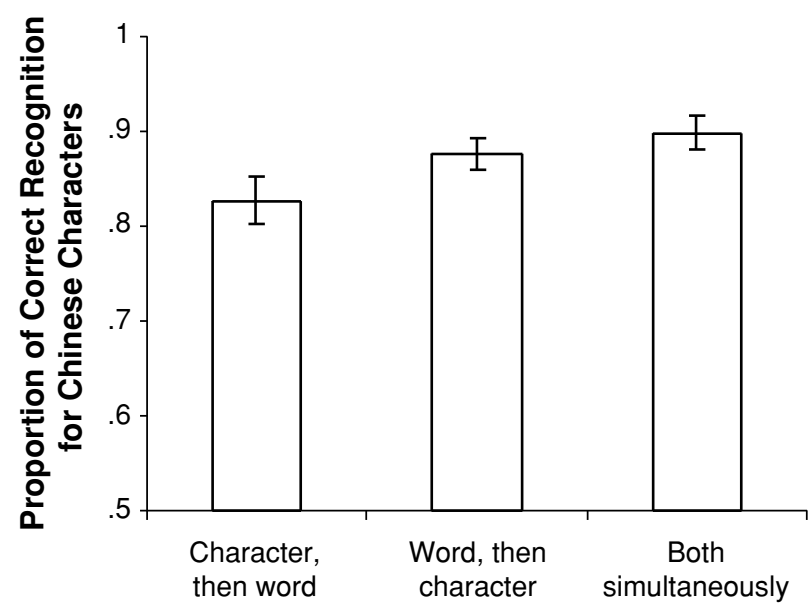

Figure 4. Results from Experiment 2. Order of presentation mattered: Showing first the word and then the character led to performance equivalent to presenting both simultaneously; performance was lower when the character was shown first and then the word. Error bars denote $\pm 1 S E$.

\section{Discussion}

The results show that the association between word and character was asymmetric. Showing the word first and then the character yielded a level of recognition ( $88 \%$ correct) that was higher than when first the character and then the word was presented ( $83 \%$ correct). This asymmetry is what was predicted from the semantic associates hypothesis, and it goes against a distinctive feature hypothesis. Thus, we conclude that subjects actively search the Chinese characters for features that can be associated with the object denoted by the word, rather than searching for distinctive features. Interestingly, recognition performance in the sequential first-word-then-character condition was statistically equivalent with performance in the simultaneous condition ( $90 \%$ correct). Note, then, that this associative process seems to be different from processes operating between pairs of words, where associations are indeed symmetric (Kahana, 2002).

Again, order of presentation within lists did not yield significant effects, indicating that learning or interference effects were minimal or canceled each other out.

\section{EXPERIMENT 3}

What is the nature of the associations that are being formed? So far, we have used labels that are very familiar and highly concrete and imageable (like Crowder, 1976, we will use those two terms interchangeably; see also note 1) - words denoting mostly common objects and living things. Either of the two factors might contribute to a better memory. Dual-trace theory (Paivio, 1971) predicts that concrete words lead to both a verbal code and a visual image. The visual image helps with building associations that can function as reliable retrieval plans. Therefore, in stimulus-response pairs, stimuli that are easy to imagine should lead to enhanced memory performance, and this is indeed what has generally been found (e.g., Bower, 1972; Paivio, 1965). Meaningfulness and familiarity have also been found to enhance performance in paired-associate paradigms (e.g., Underwood, 1964), but there are indications that the effects are situated mostly on the response side; that is, performance is boosted especially when the second term of the pair is meaningful or familiar, as opposed to when it is not (e.g., Sheffield, 1946; cited in Crowder, 1976). Meaningfulness and familiarity do have an influence on the stimulus side, because meaningful and familiar words presumably have a wider net of associative connections in semantic and/or episodic memory (e.g., Mandler, 1980). To examine the relative influence of the two factors, we tested memory for four different lists - that is, all four combinations of high and low familiarity and high and low imageability. If the associations are based primarily on visual imagery, we would expect recognition to be better if the words are easy to imagine; if the associations are based primarily on the ready availability of associative material, we would expect recognition to be better if the words are highly familiar. Given that the Chinese characters themselves are such clearly visual stimuli, one might expect a bias toward the visual in associations. 


\section{Method}

Subjects. The subjects were 22 undergraduate students, who received course credit for their participation.

Materials and Procedure. The stimulus pool for Chinese characters was identical to the pool used in Experiments 1 and 2. Four types of word lists were constructed, each type comprising two lists of 24 words. All the words were nouns and between three and five letters long. The first type consisted of nouns high on imageability and familiarity (e.g., clown and lion); the second consisted of nouns high on imageability and low on familiarity (e.g., noose and spire); the third consisted of nouns low on imageability, high on familiarity (e.g., envy and unit); and the fourth type consisted of nouns that were low on both imageability and familiarity (e.g., debut and dogma). Imageability and familiarity ratings were obtained from the MRC Psycholinguistic Database, described above. Words with high imageability rated between 500 and 626 on the 700-point imageability scale $(M=549, S D=32)$. Words with low imageability ratings rated between 300 and 400 on the 700-point imageability scale $(M=$ $362, S D=25) .{ }^{1}$ Words with high familiarity ratings rated between 500 and 600 on the 700-point familiarity scale $(M=546, S D=27)$. Words with low familiarity ratings rated between 300 and 460 on the 700 -point familiarity scale $(M=380, S D=34)$. The four types of lists were presented in a within-subjects design; order of list type was counterbalanced across subjects. Details of list and stimulus presentation were identical to those in Experiment 1.

Immediately after presentation of each list, recognition trials began. Two stimuli, one target and one foil, were presented simultaneously on the screen, side by side, and the subjects responded by pressing a key (either $\mathrm{z}$ or $/$ ) that corresponded to the location of the stimulus they recognized as having been presented during encoding; the corresponding word was projected directly above the two characters.

The subjects were tested individually in a quiet, comfortable, light-sealed room with controlled ambient lighting. All assessments were taken in a single session, typically lasting about $40 \mathrm{~min}$. The subjects were encouraged to take breaks whenever they felt tired; few subjects, however, chose to take breaks.

\section{Results}

The results are depicted in Figure 5. All the conditions yielded recognition performance significantly better than chance (i.e., $50 \%$ accuracy). An ANOVA with imageability and familiarity of the words accompanying the Chinese characters and order of presentation within conditions as within-subjects variables yielded a significant main effect of imageability $\left[F(1,21)=10.96, M S_{\mathrm{e}}=0.008\right]$; words with higher imageability values yielded better recognition. Familiarity did not influence recognition $[F(1,21)=1.90$, $\left.M S_{\mathrm{e}}=0.006\right]$, but this lack of a main effect was qualified by a familiarity $\times$ imageability interaction $[F(1,21)=$ $5.44, M S_{\mathrm{e}}=0.003$, indicating that familiarity did have an effect when the words were highly imageable, but not when the words had a low imageability value. Neither the main effect of order $\left[F(1,21)=1.02, M S_{\mathrm{e}}=0.006\right]$ nor the triple interaction $\left[F(1,21)=0.66, M S_{\mathrm{e}}=0.004\right]$ was significant, but order did interact with imageability $[F(1,21)=5.44$, $\left.M S_{\mathrm{e}}=0.006\right]$. For high-imageability words, recognition accuracy was higher on the first list presented than on the second ( $88 \%$ vs. $84 \%$ ); no such effect was observed for low-imageability words ( $81 \%$ vs. $82 \%)$.

\section{Discussion}

As was expected, Chinese characters paired with words that were easily imageable were better remembered than

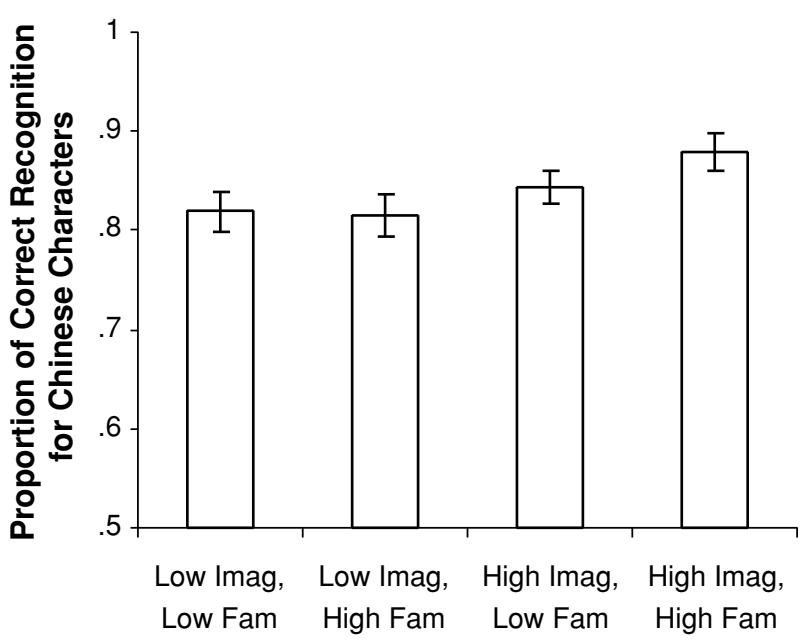

Figure 5. Results from Experiment 3. Chinese characters were recognized better when paired with highly imageable words; high familiarity further enhanced this effect. Error bars denote $\pm 1 S E$.

Chinese characters paired with words that were more difficult to form a mental picture of. Familiarity per se did not lead to better recognition, but it did have an additional augmenting effect in highly imageable words. Therefore, it appears that the associations built during the assimilation process are primarily visual in nature; the visual associations can further benefit from the word's familiarity. The latter result may occur because more familiar words have a wider network of associations with other words or with events stored in episodic memory (Mandler, 1980). The results also suggest that associations with words of high imageability lead to interference, as indicated by higher recognition for the first list presented than for the second list presented. Low-imageability words did not show this effect. The reason for this interaction is unclear.

\section{EXPERIMENT 4}

In the last experiment, we examined the time course of the assimilation process by varying the presentation time of the stimuli. We contrasted recognition memory for the combination of Chinese characters and words and for Chinese characters presented in isolation. This experiment should provide further evidence for the assimilation hypothesis. We know from earlier research (Verhaeghen et al., 2000; see Figure 1) that memory traces for Chinese characters in isolation and memory traces for words in isolation are formed very quickly; that is, performance rises above the measurement floor at very low presentation times. If our subjects truly build associations, one would expect two effects on the time-accuracy curve for the combination of words and characters. First, we would expect a delay in liftoff of the curve, because forming an association requires additional processes before a memory trace can be formed - notably, reading and interpreting the word, extraction of a relevant feature from the Chinese 
character, and the formation of an association. Second, we might expect a slower buildup of the curve, because the process of extracting features and assimilating them with the label might be slower than the less effective encoding processes used in the character-only version of the task (similar slowing effects with powerful visual mnemonics have been demonstrated before; Verhaeghen \& Kliegl, 2000).

\section{Method}

Subjects. The subjects were 31 undergraduate students, who received course credit for their participation.

Materials and Procedure. The stimulus pool in the present experiment was identical to the pool used in Experiments 1-3. Two conditions were used in a within-subjects design. The first condition entailed presentation of Chinese characters only; the second entailed the pairing of each character with a word. Each list contained 19 characters or character-word pairs.

The 19 study items of a list were presented sequentially, all at the same rate. The first list in each condition was presented at $800 \mathrm{msec} /$ word, the second at $400 \mathrm{msec} /$ word, the third at $1,600 \mathrm{msec} /$ word, the fourth at $200 \mathrm{msec} /$ word, and the final list at 3,200 msec/word. Presentation of each list started with a keypress initiated by the subject.

Immediately after presentation of each list, recognition trials began. Two stimuli, one target and one foil, were presented simultaneously on the screen, side by side, and the subjects responded by pressing a key (either $z$ or $/$ ) that corresponded to the location of the stimulus they recognized as having been presented during encoding; the corresponding word was projected directly above the two characters. The order of presentation of the stimuli within each list during retrieval did not correspond to the order of presentation within the list during encoding.

The subjects were tested individually in a quiet, comfortable, light-sealed room with controlled ambient lighting. All assessments were taken in a single session, typically lasting about $40 \mathrm{~min}$. The subjects were encouraged to take breaks whenever they felt tired; few subjects, however, chose to take breaks.

\section{Results}

The results are depicted in Figure 6A. An ANOVA with condition and presentation time as within-subjects variables yielded a significant effect of presentation time $\left[F(4,27)=53.84, M S_{\mathrm{e}}=0.017\right.$; correct recognition increased with presentation time] but not of condition $\left[F(1,30)=0.74, M S_{\mathrm{e}}=0.016\right]$. The absence of a condition main effect was qualified by a condition $\times$ presentation time interaction $\left[F(4,27)=9.36, M S_{\mathrm{e}}=0.013\right]$. As can be seen in the figure, performance in the character-only condition was superior to performance in the characterplus-word condition at short presentation times but was inferior at long presentation times, with a crossover situated somewhere between 800 and 1,600 msec.

Figure $6 \mathrm{~B}$ presents the same results, with percentage of correct recognition plotted as a function of $\log$ (presentation time). This technique has been promoted by Kahana and Loftus (1999, p. 361) to examine horizontal differences between time-accuracy curves. Briefly, if the two curves are parallel when plotted as a function of $\log$ (presentation time), this implies a constant-ratio difference between the two; that is, it implies that one curve is simply a slower version of the other curve. As can be seen in Figure 6B, the slopes for the two lines differ considerably $[t(30)=5.78]$
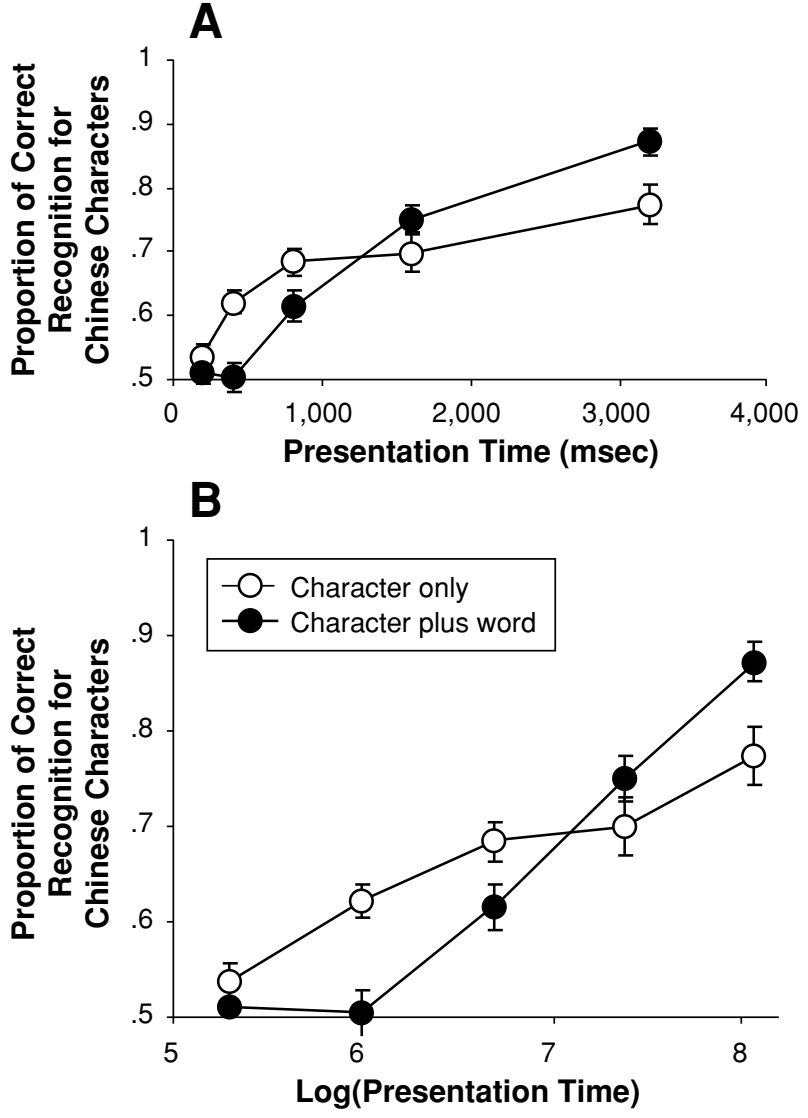

Figure 6. Results from Experiment 4. Time-accuracy functions for Chinese characters only, or characters paired with words, in (A) presentation time and (B) $\log$ (presentation time) coordinates. Presenting a word alongside the character slows down encoding but raises the asymptote. Error bars denote $\pm 1 S E$.

(slopes for the character-plus-word lines for the individual subjects were computed using only the longest four presentation times, to take the liftoff into account; the slopes were $0.08[S D=0.06]$ and $0.18[S D=0.07]$ for characteronly and character-plus-word conditions, respectively).

\section{Discussion}

The time-accuracy curves for the character-only and the character-plus-word conditions show different characteristics. A first difference is situated in the liftoff from the measurement floor. The former curve resulted in the almost immediate formation of a measurable memory trace; this replicates the findings from the corresponding condition in Verhaeghen et al. (2000). The latter curve, however, was noticeably delayed; the subjects needed more than $400 \mathrm{msec}$ before a trace was formed. The delay in liftoff of the curve for the latter condition strongly suggests that the subjects indeed formed mental associations that presupposed a number of processes before a memory trace could be formed. These processes could include reading and encoding the word and an active search for relevant features in the Chinese character. 
Second, after logarithmic transformation of the presentation time axis, the slope in the character-plus-word condition is much steeper than the slope in the character-only condition. This result indicates that the memory-enhancing effect of the word-character associations goes beyond rescaling; that is, it is more than a simple speeding-up of the encoding processes in the character-only condition. The difference in slope could be due to either of two mechanisms - namely, (1) slower processing in the characteronly condition than in the character-plus-word condition or (2) a difference in asymptotic accuracy between the two conditions. We deem the latter option the more likely: The linear rise in $\log$ (presentation time) for the characterplus-word condition suggests that the asymptote may be situated at $100 \%$ correct recognition; the curve for the character-only condition, on the other hand, seems to be negatively accelerated in log-transformed space, indicating that it will never reach $100 \%$ correct recognition (the latter result was also obtained by Verhaeghen et al., 2000). If we accept this position, it seems that the assimilation process indeed slows down processing considerably: Inspection of Figure 6A suggests that the asymptote will be reached much later in the character-plus-word condition than in the character-only condition.

If we further compare the results from Experiment 4 with the results of Verhaeghen et al. (2000, here reproduced in Figure 1), we find that the results for the character-plus-word condition fall in between those for the word-only and the character-only conditions; that is, we find the aforementioned delay and then a slow rise (in comparison with either of the other two conditions) to an asymptote that is comparable to (if not higher than) the asymptote in the word-only condition in the previous experiment. (Note that the methodology of both experiments was identical in terms of number of items per list, the appearance of the stimuli on the screen, and the recognition procedure.) Thus, it seems that the assimilation process boosts recognition to about the level that the word by itself would yield, provided that the associated word is shown during the retrieval phase. At the same time, the assimilation process proceeds at a slower pace than the processes that make for successful encoding of a word alone.

\section{GENERAL DISCUSSION}

In this study, we successfully enhanced recognition memory for Chinese characters by providing a verbal label along with the character. Although the subjects were not explicitly instructed to associate the word with the character, this seems to be what they did. Evidence for the presence of an assimilative association process arises out of three findings. The first is that the effect operates only when the word is presented not just at encoding, but also during the retrieval phase (Experiment 1). This suggests that the word acts as a direct associative cue for the character, rather than merely as an aid for the extraction of memorable features from the Chinese character. The second relevant finding is that showing first the word and then the character yields results that are indistinguish- able from showing both simultaneously but that showing the character first does lead to a decrease in recognition memory performance. This suggests that subjects first read the word and decode its meaning and then search for features in the character that can be associated with the word's meaning, rather than extracting defining features from the character and associating those with the word (Experiment 2). The third relevant finding is that the presence of the word during encoding alters the dynamics of the time-accuracy function-namely, by delaying and slowing the buildup of the memory trace (Experiment 4). This suggests that the encoding processes are delayed when a label is present - presumably, because reading the word, extracting its meaning, and choosing the character features that match the meaning are time-consuming prerequisites for encoding. It also suggests that the encoding processes when the label is present are slower than the processes usually applied to memory tasks (words or characters). This finding suggests the deployment of an additional process, which might well be the pairing of the semantic features from the word with the visual features from the character. In addition, Experiment 3 demonstrated that the associations are primarily visual in nature, because words with high imagery values resulted in better recognition for the associated Chinese characters. Breadth of associability (operationalized as familiarity) played an additional role, but only if the word was highly imageable. These findings support the Gestalt-oriented integration view of labeling effects and goes against theories that propose that the effect of verbal labeling resides merely in enhanced encoding.

\section{REFERENCES}

Bellezza, F. S. (1983). Mnemonic-device instruction with adults. In M. Pressley \& J. R. Levin (Eds.), Cognitive strategy research: Psychological foundations (pp. 51-73). New York: Springer.

Bower, G. H. (1972). Mental imagery and associative learning. In L. W. Gregg (Ed.), Cognition in learning and memory (pp. 51-88). New York: Wiley.

Carmichael, L., Hogan, H. P., \& Walter, A. A. (1932). An experimental study of the effect of language on the reproduction of visually perceived forms. Journal of Experimental Psychology, 15, 73-86.

Craik, F. I. M., \& Tulving, E. (1975). Depth of processing and the retention of words in episodic memory. Journal of Experimental Psychology: General, 104, 268-294.

Crowder, R. G. (1976). Principles of learning and memory. Hillsdale, NJ: Erlbaum.

Daniel, T. C., \& Toglia, M. P. (1976). Recognition gradients for random shapes following distinctive or equivalent verbal association training. Journal of Experimental Psychology: Human Learning \& Memory, 2, 467-474.

ELLIS, H. C. (1968). Transfer of stimulus predifferentiation to shape recognition and identification learning: Role of properties of verbal labels. Journal of Experimental Psychology, 78, 401-409.

ELLIS, H. C. (1973). Stimulus encoding processes in human learning and memory. In G. H. Bower (Ed.), The psychology of learning and motivation: Advances in research and theory (Vol. 7, pp. 123-182). New York: Academic Press.

Gilhooly, K. J., \& LoGIE, R. H. (1980). Age-of-acquisition, imagery, concreteness, familiarity, and ambiguity measures for 1,944 words. Behavior Research Methods \& Instrumentation, 12, 395-427.

Kahana, M. J. (2002). Associative symmetry and memory theory. Memory \& Cognition, 30, 823-840.

Kahana, M. J., \& Loftus, G. (1999). Response time versus accuracy 
in human memory. In R. J. Sternberg (Ed.), The nature of cognition (pp. 322-384). Cambridge, MA: MIT Press.

MANDLER, G. (1980). Recognizing: The judgment of previous occurrence. Psychological Review, 87, 252-271.

Meissner, C. A., \& Brigham, J. C. (2001). A meta-analysis of the verbal overshadowing effect in face identification. Applied Cognitive Psychology, 15, 603-616.

PAIVIO, A. (1965). Abstractness, imagery, and meaningfulness in pairedassociate learning. Journal of Verbal Learning \& Verbal Behavior, $\mathbf{4}$, 32-38.

PaIvIO, A. (1971). Imagery and verbal processes. Hillsdale, NJ: Erlbaum.

Paivio, A., Yuille, J. C., \& Madigan, S. A. (1968). Concreteness, imagery, and meaningfulness values for 925 nouns. Journal of Experimental Psychology Monographs, 76(1, Pt. 2), 1-25.

Ranken, H. B. (1963). Language and thinking: Positive and negative effects of naming. Science, 141, 48-50.

SANTA, J. L. (1975). Verbal coding and redintegrative memory for shapes. Journal of Experimental Psychology: Human Learning \& Memory, 104, 286-294.

Toglia, M. P., \& Battig, W. R. (1978). Handbook of semantic word norms. Hillsdale, NJ: Erlbaum.

Tulving, E., \& Thomson, D. M. (1973). Encoding specificity and retrieval processes in episodic memory. Psychological Review, 80, $352-373$

UNDERwood, B. J. (1964). Degree of learning and the measurement of forgetting. Journal of Verbal Learning \& Verbal Behavior, 3, 112129.

Verhaeghen, P., \& Kliegl, R. (2000). The effects of learning a new algorithm on asymptotic accuracy and execution speed in old age: A reanalysis. Psychology \& Aging, 15, 648-656.

Verhaeghen, P., Marcoen, A., \& Goossens, L. (1992). Improving memory performance in the aged through mnemonic training: A meta-analytic study. Psychology \& Aging, 7, 242-251.

Verhaeghen, P., Palfai, T., Cerella, J., Buchler, N., Johnson, M. P., D'Eredita, M., et AL. (2000). Age-related dissociations in time-accuracy functions for recognition memory: Utilizing semantic support versus building new representations. Aging, Neuropsychology, \& Cognition, 7, 260-272.

\section{NOTE}

1. One of the reviewers suggested that words should have been selected on the basis of concreteness, rather than imageability. To examine the consequence of our choice, we retrieved the concreteness value for each of the words in our lists (96 words low on imageability and 96 words high on imageability). The correlation between the imageability values and concreteness values was .90, suggesting that, in practice, the two types of ratings denoted essentially the same characteristic. The average concreteness value in the high-imageability words was $569(S D=$ 40 ); in the low-imageability group, the average concreteness value was $350(S D=63)$. Therefore, the average of the two word samples was 4.2 $S D$ s apart on concreteness, in comparison with a distance of $6.4 S D$ s on imageability.

(Manuscript received April 26, 2004; revision accepted for publication May 18, 2005.) 